\author{
M. Landowski, M. Budzik, K. Imielińska* \\ Gdansk University of Technology, Faculty of Mechanical Enginering, Narutowicza 11/12, \\ 80-233 Gdansk, Poland, *kimielin@pg.gda.pl
}

\title{
WATER SORPTION AND BLISTERING OF GFRP LAMINATES WITH VARYING STRUCTURES
}

\begin{abstract}
The microstructures, water absorption as well as blistering was studied for bi- and tri- axial glass fibre vinyl ester and polyester -matrix laminates coated with gel coat layer and uncoated. The effect of manufacturing technique on water sorption characteristics was considered. It was found that water sorption characteristics of GFRP depend on manufacturing methods. The least water intake was found for specimens having perfect microstructure due to manufacturing by infusion process. Water sorption saturation value was reached after ca. 50 days of accelerated test at $70^{\circ} \mathrm{C}$ and was found to correspond to 250 days conditioning at $20^{\circ} \mathrm{C}$. Blistering evolution was illustrated and SEM images shown corresponding to degradation of the gel coat layer and the laminate during the exposure in water.
\end{abstract}

Keywords: Polymer composites, durability, scanning electron microscopy (SEM)

\section{INTRODUCTION}

Glass/polyester laminates have been used in boat building industry for over 50 years now due to their advantages such as low density and low manufacturing costs. However, the surface of the glass fibre reinforced composites coated with gel coat layer is in permanent contact with water which causes water absorption and blistering of the laminate surface in longer exposure times [1-3].

When first blisters are found on the surface of the protective coating the laminate is already affected by water which causes irreversible damage in the structure of the material. Blistering and degradation of the laminates in water is caused mainly by osmosis. This issue was widely discussed in the past [4,5]. Water molecules keep osmosing through the laminate, and join the chemicals in the bubble, steadily building up hydraulic pressure. Eventually this causes the surface of the moulding to blister. When the surface is full of blisters the stage of degradation is reached when the diffusing water reaches internal layers of the laminate thus causing reduction in the interlaminar adhesion of the material [5].

In the recent years new types of bi-axial and tri-axial glass fibre fabrics were developed to cut down the labour cost in the marine industry. These types of reinforcements were designed in particular for the advanced manufacturing methods such as infusion in which layers of dry fibre fabrics are laid in the mould and are impregnated by the resin by vacuum process. However, nowadays theses textiles are often used by boat builders employing simple hand lay-up method which may lead to poor impregnation of the fibres. Accordingly, the question raised in this study was about the effect of manufacturing technique and the resulting 
structural quality of the materials with multilayer reinforcement on their water uptake and blistering characteristics.

\section{MATERIALS AND EXPERIMENTS}

Five typical marine laminate plates were fabricated at motor boat building plants in Gdańsk, Poland: Galeon, (Laminates 1-4) and at HTEP (Sunreef Yachts) (Laminate 5). Laminates 1-3 were uncoated. The manufacturing methods were: hand lay-up (Laminate 1) vacuum bagging (Laminate 2) and infusion (Laminate 3). The matrix resin 1 was unsaturated pre-accelerated vinyl ester urethane resin BUFA Atlac 580 ACT, (DSM Composite Resins). Catalyst was $1,5 \%$ Metox 50. Specimens were cured at room temperature for 24 hours. High performance (HP), glass fibers textiles were used: two plies of tri-axial fabric (chopped strand mat CSM $500 \mathrm{~g} / \mathrm{m}^{2}$ stitched with a $0 / 90^{\circ}$ unidirectional UD fabric 860 $\mathrm{g} / \mathrm{m}^{2}$ ). Laminate thicknesses obtained were respectively: $3,8 \mathrm{~mm}, 2,6 \mathrm{~mm} \mathrm{2,7} \mathrm{mm}$. SEM micrograph of the layered structure of tri-axial HP textile is illustrated in Fig.1.
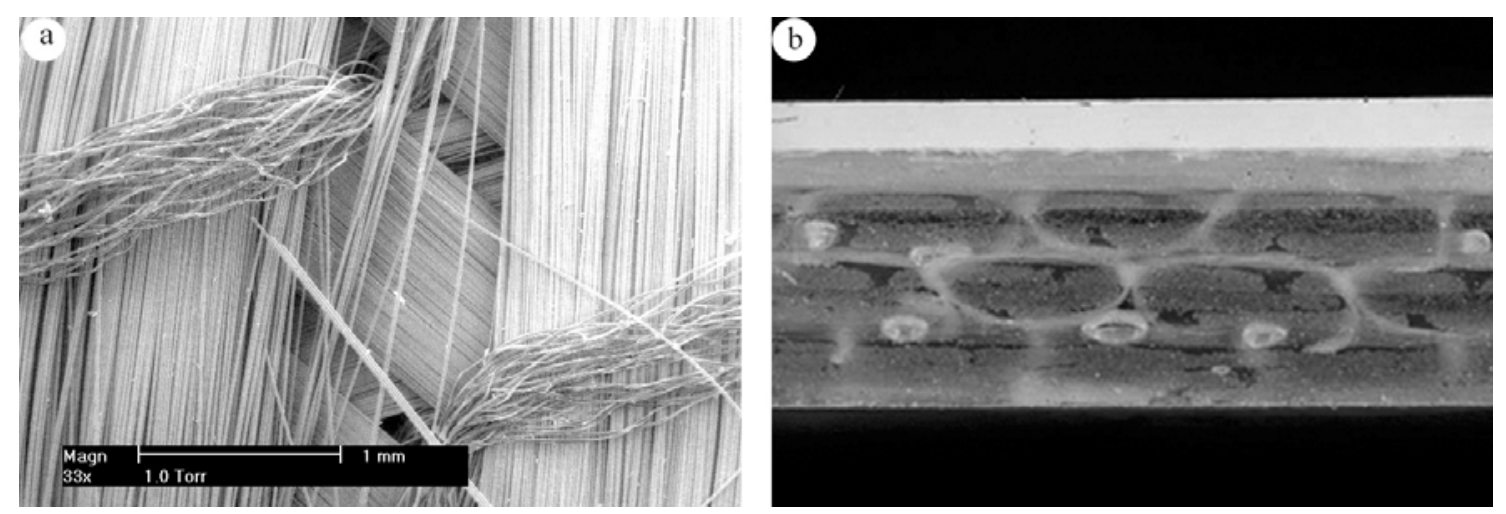

Fig. 1. a) SEM micrograph showing layered structure of tri-axial $\left(0 \%-45^{\circ} \%+45^{\circ}\right)$, glass fibre textile used in the fabrication of laminate hulls, b) Laminate 4 plate: perpendicular white lines show stitching of the fabrics using polyester thread

Laminate 4 was coated with isophtalic polyester gel coat LS 30PA (Scott Bader) (thickness $0,5 \mathrm{~mm}$ ). The matrix 2 was unsaturated polyester resin Crystic 701PA. The reinforcement HP biaxial glass cloth (CSM $110 \mathrm{~g} / \mathrm{m}^{2} / 45^{\circ}$ fabric), biaxial glass cloth (CSM $861 \mathrm{~g} / \mathrm{m}^{2}$ and $0^{\circ} / 90^{\circ}$ fabric). Dry fibers were impregnated by the resin using infusion method. Laminate thickness was $2,7 \mathrm{~mm}[7]$.

Laminate 5 was coated with isophtalic Polycolor ISO BR Blank 9199 gel coat of variable thickness: 1,5 mm, 2,3 mm and $3 \mathrm{~mm}$. The unsaturated polyester resin (matrix 3) Cray Valley Norsodyne S 21150 TAE supplied by Cray Valley was used to impregnate the fibres using hand lay-up method. Catalyst used to cure both the resin and the gel coat was Curox M-300 supplied by Degussa Initiators. The Glass fiber reinforcement stacking sequence was typical of boat hulls: chopped strand matt $(\mathrm{CSM})\left(150 \mathrm{~g} / \mathrm{m}^{2}\right) /$, CSM $\left(450 \mathrm{~g} / \mathrm{m}^{2}\right) /$, CSM $\left(150 \mathrm{~g} / \mathrm{m}^{2}\right) /$ biaxial fabric $\left(816 \mathrm{~g} / \mathrm{m}^{2}\right) /$ tri-axial fabric $\left(868 \mathrm{~g} / \mathrm{m}^{2}\right)$. Chopped strand mats were supplied by Krossglass SA 50. Biaxial (HPT 810-E45/3BED1-SF090) and tri-axial (HPT 860- 
E45/2BCD1-SF060) fabrics were HPT (high performance textiles) supplied by F.A. Kumpers. Laminate thickness (without gel coat layer) was $5 \mathrm{~mm}$.

Laminate plates were cut into specimens $45 \times 15 \mathrm{~mm}$ for water absorption tests. The edges were painted with epoxy resin to prevent fast water sorption through the edges.

Laminate 5 surface (gel coat thickness 1,5 mm) was ground using emery paper 600, 1500 and polished using $\mathrm{Al}_{2} \mathrm{O}_{3}(3 \mu \mathrm{m})$ to obtain gel coat thickness $0,5 \mathrm{~mm}$.

Water sorption accelerated tests were made based on the ASTM standard [8] by immersion in a vessel at $70^{\circ} \mathrm{C}$ for up to 50 days, which corresponds to the exposure of 30 years at $19^{\circ} \mathrm{C}$ [10]. Laminate 5 was subjected to water immersion tests at $50^{\circ} \mathrm{C}$ and $20^{\circ} \mathrm{C}$ to compare the water sorption rate in accelerated and natural conditions. The mass of the specimens was tested every 5-10 days using precision scales RATWAG WPA 180/C/1 (0,1 mg precision).

The blisters formation in Laminates 4 and 5 was studied by estimation of incubation time for the first blister to appear at the centre $(30 \mathrm{~mm} \times 8 \mathrm{~mm})$ rectangle. The cross sections of coated and uncoated specimen types were made across the blistered area in order to assess laminates quality (voids, resin pockets, impregnation of the fibres) and to observe the subsurface damage, corresponding to the surface blisters. Samples were ground on emery papers, polished using $\mathrm{Al}_{2} \mathrm{O}_{3}$ powder $(3 \mu \mathrm{m})$ and micrographs were taken using scanning electron microscope Philips-FEI XL 30 ESEM.

\section{RESULTS AND DISCUSSION}

\section{Microstructure}

Figs. 2a-c show the SEM micrographs of laminate unconditioned manufactured by: hand lay-up (Fig. 2a), vacuum bagging (Fig. 2b), infusion (Fig. 2c). Typical features observed in Fig. $2 \mathrm{a}$ are: extensive resin- rich areas and large voids spaces. The high performance bi-axial and tri-axial stitched textiles are well impregnated by the resin. Vacuum bagging specimen appears to be excessively compacted by the pressure which lead to reduction in resin content and poor impregnation of the fibres (dry spots) resulting in weak matrix/fibre interfacial bonding. Water may diffuse along such interfaces through capillary action causing debondings and interfacial cracks [1]. This may explain the abnormally fast water sorption observed for vacuum bagging- samples. Infusion-specimen (Fig. 2c) is very compact (no resin- rich areas which are regarded as structural defect) are observed in the material, However fibres are well impregnated by the resin (no dry spots). Water sorption in such specimens is very low, below $1 \%$. The micro-structure appears to be perfect. 

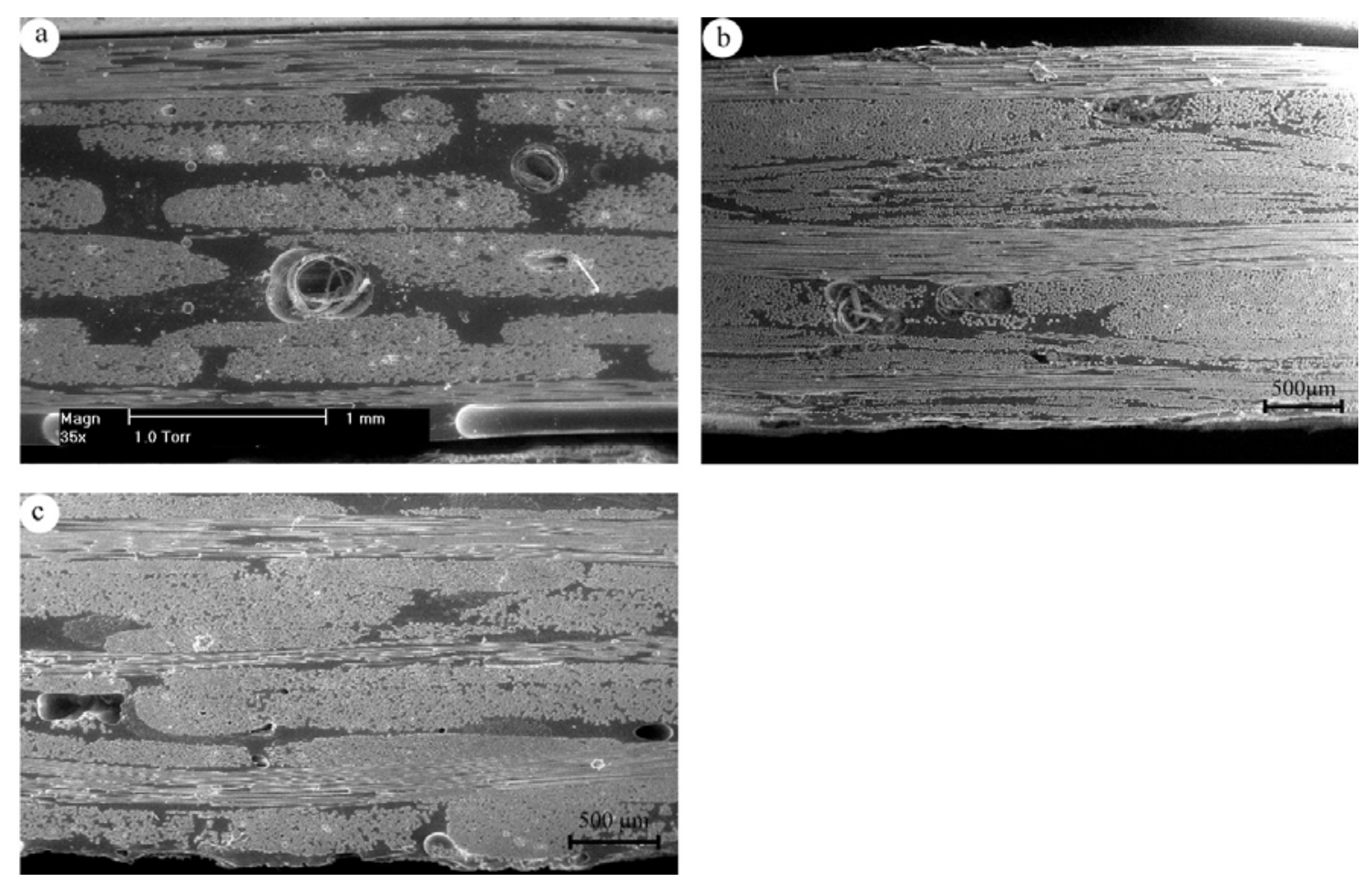

Fig. 2. SEM micrographs of laminates1-3 unconditioned manufactured by: a) laminate 1- hand lay-up, b) laminate 2 - vacuum bagging, c) laminate 3 - infusion

\section{Water sorption characteristics}

Fig. 3a illustrates water sorption characteristics typical of of vinylester urethane -matrix Laminates 1-3 (L1-3) conditioned at $70^{\circ} \mathrm{C}$. Laminate fabricated using vacuum bagging absorbs much more water than the other two. The least water intake was for Laminate 3 manufactured using infusion process. Very low water absorption saturation value is obtained (Fig. 3b) for coated polyester-matrix laminate L4 protected by thin, $0,5 \mathrm{~mm}$ polyester gel coat, (fabricated using infusion method), compared to the results obtained for laminate 4 fabricated by hand lay-up (coated with $0,5 \mathrm{~mm}$ gel coat). Fig. $3 \mathrm{~b}$ also shows that the increased gel coat thickness $(3 \mathrm{~mm})$ provides better protection in terms of water absorption than the thin gel coat layer. However, it has been observed that thick gelcoat layer when blistered tends to fracture more easily than the thin layer.
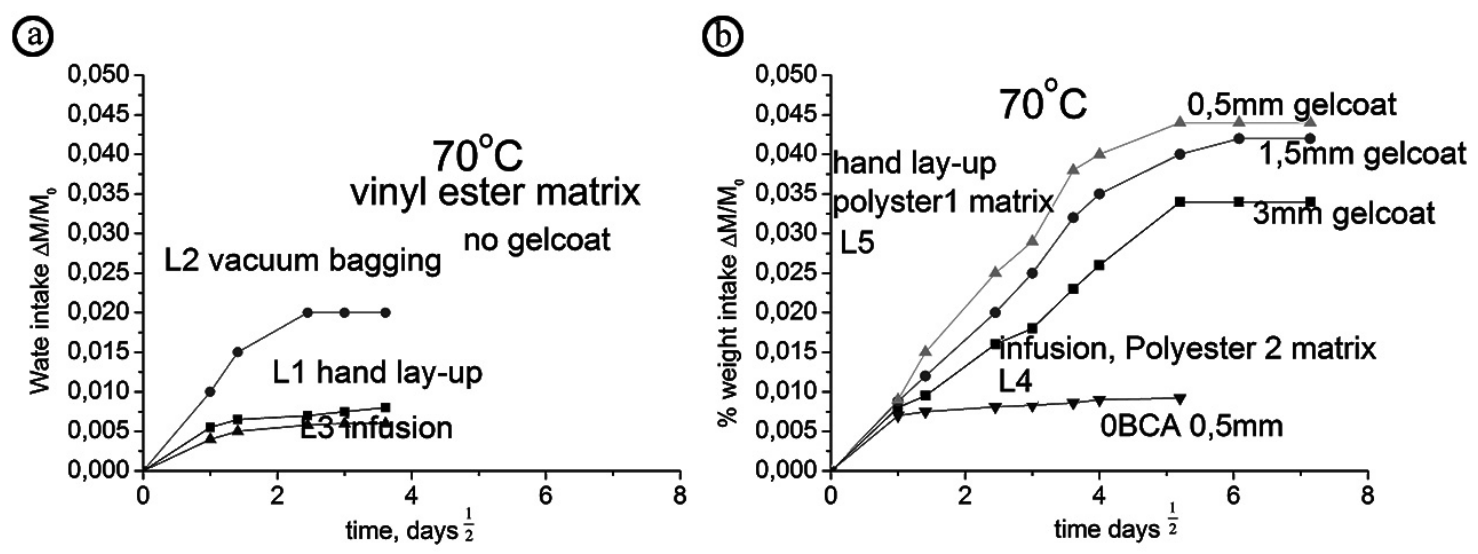

Fig. 3. Water absorption characteristics at $70^{\circ} \mathrm{C}$ of a) uncoated Laminates 1-3 (L1-3), b) coated Laminates 4,5 $(\mathrm{L} 4, \mathrm{~L} 5)$ with variable gelcoat thickness and manufacturing methods 
The results of accelerated test at $70^{\circ} \mathrm{C}$ are not very realistic for boat hull laminates immersed in water. Accordingly, it was interesting to study how these characteristics correspond to the results of the tests made at lower temperatures: $50^{\circ} \mathrm{C}$ and especially $20^{\circ} \mathrm{C}$. The comparative tests results are illustrated in Fig 4 showing water sorption characteristics of polyester matrix laminate 5 coated with $0,5 \mathrm{~mm}$ gelcoat layer and manufactured using infusion process, conditioned at $70^{\circ} \mathrm{C}, 50^{\circ} \mathrm{C}$ and $20^{\circ} \mathrm{C}$.

In over a year long test at $20^{\circ} \mathrm{C}$ the saturation value of $1 \%$ absorbed water was reached. To be noted is that after 50 days exposure at $70^{\circ} \mathrm{C}$ the saturation values obtained in the accellerated test almost reached the $20^{\circ} \mathrm{C}$ saturation value. At $50^{\circ} \mathrm{C}$ more time is needed to reach the saturation value. However, the tests were interrupted after 50 days.

\section{Blistering behaviour}

Blisters incubation time as a function of temperature (Laminate 4) is demonstrated in Fig. 5. At $70^{\circ} \mathrm{C}$ blister incubation time is only a few days which corresponds to the saturation time on the water sorption curve (Fig. 4). Fig. 6 shows photographs illustrating the development of blisters on the gel coat surface at $70^{\circ} \mathrm{C}$.

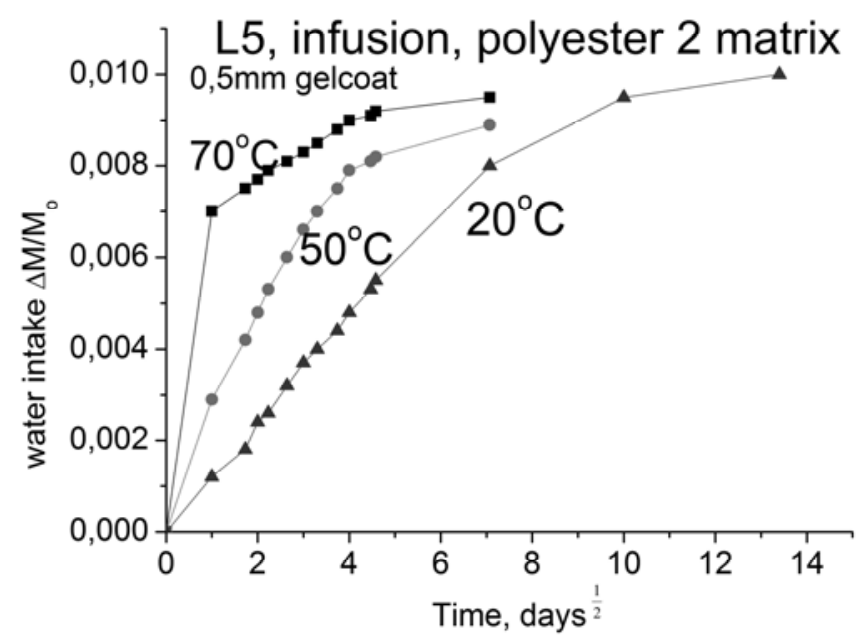

Fig. 4. Water intake vs square root of time at $20^{\circ} \mathrm{C}, 50^{\circ} \mathrm{C}, 70^{\circ} \mathrm{C}$ for $0,5 \mathrm{~mm}$ gel coat, polyester matrix (laminate 5 ) fabricated by infusion

The first, very small blisters form after 4 days exposure (laminate 5). Soon (8-10 days) all the surface is covered with large blisters, 1-2mm in diameter. By 27 days (Fig. $6 \mathrm{c}$ ) the blisters combine and some large blisters fracture. The corresponding cross section of severely blistered and fractured gel coat and laminate are illustrated in the SEM micrographs. The undamaged laminate 5 (Fig. 7a) is compared with the damaged laminate (Fig. 7b) (corresponding to Fig 6c). To be noted (Fig. 7b) are long cracks extending parallel to the gel coat layer across the laminate thickness and sub-surface cracks in the gel coat layer corresponding to surface blisters and cracks. 


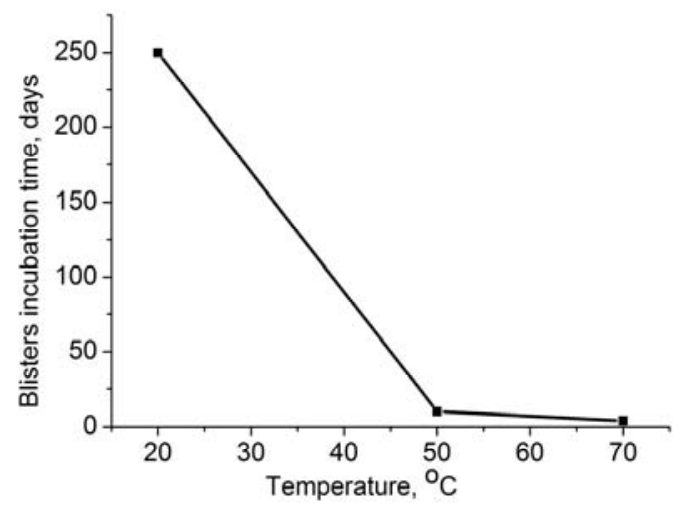

Fig. 5. Blisters incubation time for Laminate 4 at $70^{\circ} \mathrm{C}, 50^{\circ} \mathrm{C}, 20^{\circ} \mathrm{C}(0,5 \mathrm{~mm}$ gel coat thickness)
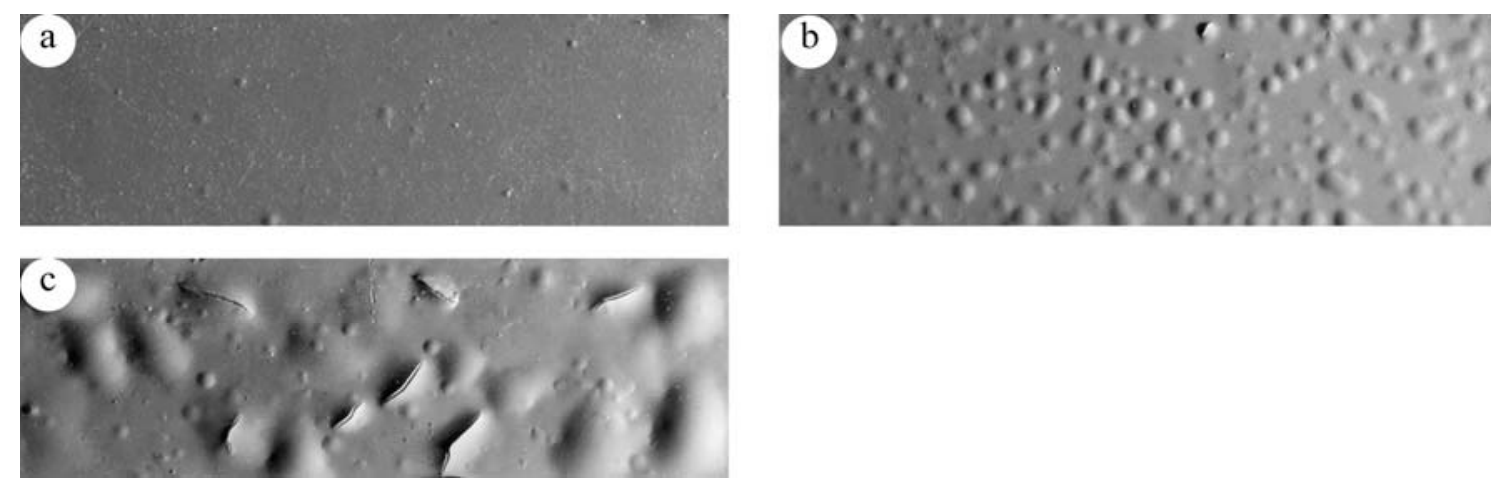

Fig. 6. Development of blisters on coated, (3mm thick gel coat) Laminate 4 and L5 with $0,5 \mathrm{~mm}$ thick gel coat: a) L4, incubation of the first blisters ( 5 days), b) L5 surface covered with blisters ( 12 days),

c) L5 cracks and large blisters after 27 days exposure
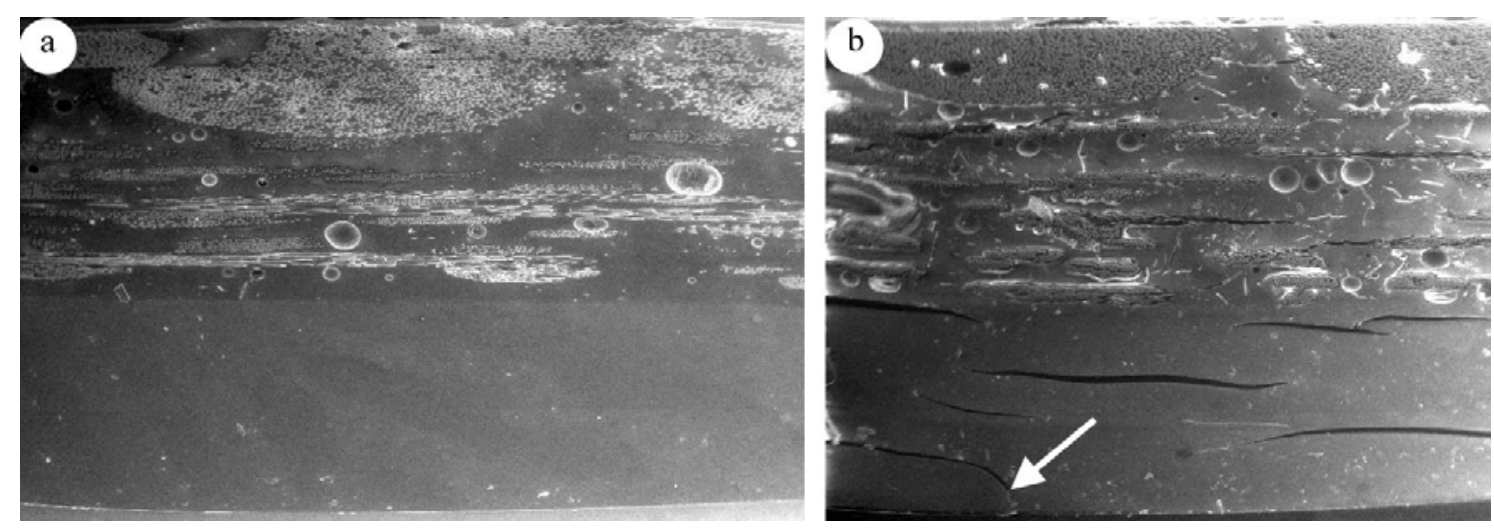

Fig. 7. a) Laminate 5 cross section before conditioning, b) microstructure of damaged laminate (L5) and sub-surface cracks in the gel coat layer corresponding to the surface blisters and cracks (arrow) developed after 27 days exposure in water at $70^{\circ} \mathrm{C}$ 


\section{CONCLUSIONS}

The microstructure, water intake as well as blistering was studied for bi- and tri- axial glass fibre vinyl ester and polyester -matrix laminates coated with gel coat layer and uncoated. The effect of manufacturing technique on water sorption characteristics was considered.

It was found that:

1. Water sorption characteristics of glass/polyester laminates reinforced with bi- and triaxial glass textiles depend on manufacturing methods.

2. The least water uptake saturation value was for samples manufactured by infusion method both for uncoated vinyl ester matrix laminate as well as coated polyester -matrix composite compared to the vacuum bagging and hand lay-up.

3. Water sorption saturation value reached after ca. 50 days of accelerated test at $70^{\circ} \mathrm{C}$ was found to correspond to 250 days conditioning at $20^{\circ} \mathrm{C}$.

4. Blisters and cracks observed on the surface of the gel coat immersed in hot water for 27 days correspond to the severe damage across the whole thickness of the laminate.

\section{ACKNOWLEDGEMENTS}

Authors wish to thank Kamil Marczyk and Michał Stempa, the former students of Gdańsk University of Technology for their contribution to this work.

\section{REFERENCES}

1. Imielińska K.: Degradation and damage of advanced laminate polymer composites due to environmental effects and low velocity impact, Wydawnictwo Politechniki Gdańskiej, Gdańsk 2005.

2. Huang G, Hongxia S., Effect of water absorption on the mechanical properties of glass polyster composites, Materials and Design 28(2007) 1647-1650.

3. Rajapakse Y.D.S., Hui D.: Marine Composites: Editorial: Composites: Part B, 35, 2004 447-450.

4. T. Rockett, V. Rose, Causes of Boat hull blistering. US. Coast Guard Grant Report 1501.83. 1987.

5. Norwood L.S., Holton E.C. : „The effect of poor interlaminar adhesion on blister formation in GRP in contact with water" Materials \& Design vol. 12, April 1991.

6. Turnbull A., Broughton W.R.: Environmental degradation of polymers and polymer composites, Proc. Conf. EDEM, 2003, Bordeaux 1-3 07. 2003.

7. Marczyk K. Master degree thesis, Gdańsk University of Technology Mechanical Faculty 2007.

8. ASTM D570-98 (2005) Standard Test Method for Water Absorption of Plastics. 